\title{
A young woman with disorientation (2010: 1a)
}

C European Society of Radiology 2009

\section{Case report}

A 34-year-old woman, originally from Brazil but living in Portugal for 5 years, was admitted for episodes of disorientation, non-specific visual changes and bilateral weakness. Neurological examination disclosed cognitive deficit in several domains and no focal deficits. Brain computed tomography showed a subcortical hypodense area in the left frontal lobe. Magnetic resonance imaging (Fig. 1), angiography (Fig. 2) and digital subtraction angiography (Fig. 3) were performed.

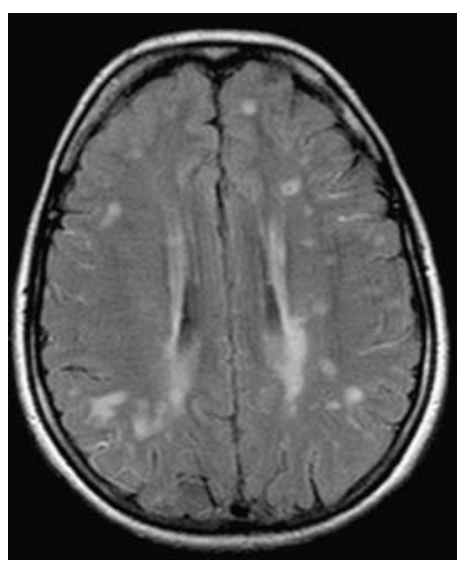

Fig. 1 Axial T2 FLAIR

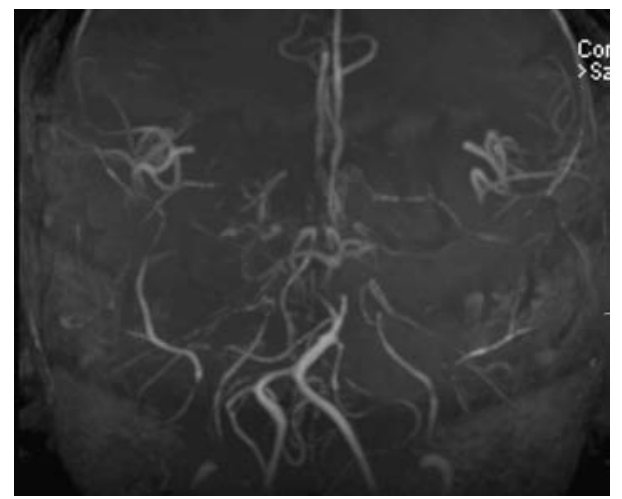

Fig. 2 Maximal intensity projection reconstruction of a threedimensional time-of-flight acquisition

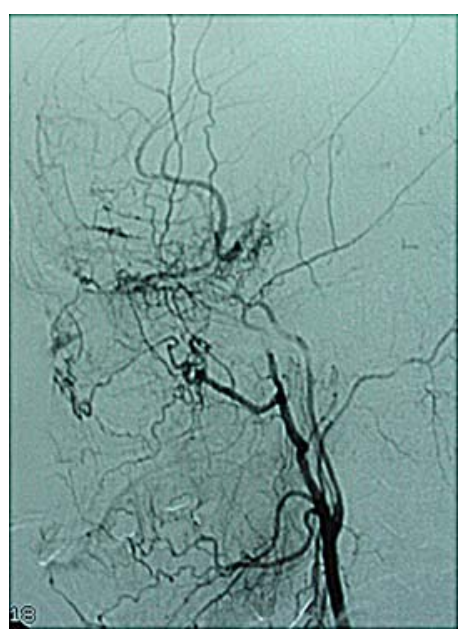

Fig. 3 Lateral view of left carotid angiogram

What is the diagnosis?

Readers are invited to supply one possible diagnosis via electronic means to: robert.hermans@uzleuven.be

The subject of the e-mail should include 'Interpretation Corner' and the number given above (e.g. Interpretation Corner 2010: 1a).

They should include their name, title, address, fax number and telephone number.

Deadline: one clear calendar month from the distribution date.

The names of the first 25 radiologists submitting the correct diagnosis will be published (only one from any individual centre and none from the host institution!).

Three months after the initial publication of the case history, the authors will publish the final diagnosis. 